\title{
APLICAÇÃO DAS FUNÇÕES DE PARTIDA E EQUAÇÕES CÚBICAS DE ESTADO NOS CICLOS TERMODINÂMICOS DE POTÊNCIA A VAPOR
}

\author{
A. M. do NASCIMENTOํㅛ P. F. ARCE-CASTILLO ${ }^{1}$ \\ ${ }^{1}$ Universidade de São Paulo, Escola de Engenharia de Lorena \\ E-mail para contato: parce@usp.br
}

\begin{abstract}
RESUMO - As aplicações das Funções de Partida e Equações Cúbicas de Estado são muito importantes na resolução de problemas envolvendo ciclos termodinâmicos, pois muitas vezes as propriedades de fluidos mais complexos utilizados nestes ciclos, como fluidos refrigerantes pouco conhecidos, não estão disponíveis em tabelas ou diagramas termodinâmicos, tornando a resolução destes problemas uma tarefa complexa. O objetivo deste trabalho é utilizar as funções de partida da entalpia e entropia na equação cúbica de estado de Peng-Robinson, para o cálculo do rendimento de um ciclo termodinâmico de potência (Rankine). Foram realizados uma série de equacionamentos, que utilizaram a equação de Peng-Robinson nas funções de partida da entalpia e entropia, e através delas podese obter a eficiência do ciclo. A realização dos cálculos foi feita usando software MS-Excel. Foram necessários dados de pressão e temperatura crítica, fator acêntrico e as constantes de calor específico do fluido para o ciclo Rankine. Para a validação dos resultados obtidos, foi utilizado um software comercial fornecido pelo ChemSOF (Equations of State Property Calculations) que fornece dados da função de partida da entalpia, entropia e fator de compressibilidade. A eficiência obtida foi comparada pela calculada através de tabelas termodinâmicas. Os resultados foram razoáveis e próximos aos apresentados através das tabelas e do software comercial.
\end{abstract}

\section{INTRODUÇÃO}

Para químicos e engenheiros no geral, o estudo das propriedades termodinâmicas são muito importantes, pois o conhecimento das propriedades das substâncias é essencial para o entendimento de processos e ciclos presentes no setor industrial. Na graduação geralmente a determinação de propriedades de substâncias puras em ciclos termodinâmicos é feita consultando tabelas e diagramas termodinâmicos. Porém na indústria muitas vezes o engenheiro encontra ciclos complexos, onde o fluido de processo é um novo refrigerante ou um gás com múltiplos componentes, para os quais não existem propriedades termodinâmicas tabeladas. Sendo assim, para a resolução de problemas envolvendo essas substâncias em ciclos termodinâmicos foi necessário recorrer à aplicação das funções de partida e equações cubicas de estado (EdE), que oferecem uma abordagem geral e que pode ser aplicável a qualquer fluido (Elliot e Lira, 2012). Também é uma vantagem utilizar as funções de partida com as equações cúbica de estado, pois diminui os erros que são trazidos por interpolações dos valores de qualquer propriedade termodinâmica quando se utilizam diagramas ou tabelas termodinâmicas na resolução de problemas. 


\subsection{Funções de partida}

Uma função de partida é calculada como a diferença entre dois estados termodinâmicos, um estado real e um estado ideal. Ela foi criada para estimar propriedades que não são mensuráveis e que não possuem valores absolutos, assim só podem ser estabelecidas pela diferença, como é o caso da entalpia, entropia, energia interna, energia livre de Helmholtz, energia livre de Gibbs. Estas propriedades são consideradas Propriedades ou Funções de Estado. Isto significa que as mudanças de seus valores dependem apenas dos estados inicial e final (Poling et al., 2001).

A entalpia e entropia são funções que dependem da temperatura $(\mathrm{T})$ e da pressão $(\mathrm{P})$, porém também podem ser expressas em função de outras propriedades como o volume $(\mathrm{V})$ e calor específico $(\mathrm{Cp})$, dependendo da convenção adotada. Considerando que um fluido muda do estado 1, que possui propriedades $\left(\mathrm{T}_{1}, \mathrm{P}_{1}\right)$, para o estado 2 que possui $\left(\mathrm{T}_{2}, \mathrm{P}_{2}\right)$, o cálculo da variação de entalpia do fluido é dado por três processos:

- Função de partida 1: calculado pela diferença de entalpia do estado real $\left(\mathrm{T}_{1}, \mathrm{P}_{1}\right)$ para o estado de gás ideal $\left(\mathrm{T}_{1}, \mathrm{P}_{1}\right)$.

- Gás Ideal: que é dado pela variação de entalpia do estado de gás ideal em $\left(\mathrm{T}_{1}, \mathrm{P}_{1}\right)$ e o estado do gás ideal em $\left(\mathrm{T}_{2}, \mathrm{P}_{2}\right)$.

- Função de partida 2: calculado pela diferença de entalpia do estado real $\left(\mathrm{T}_{2}, \mathrm{P}_{2}\right)$ para o estado de gás ideal $\left(\mathrm{T}_{2}, \mathrm{P}_{2}\right)$.

Sendo assim, o cálculo da entalpia do fluido envolvendo os três processos é dado pela Equação 1. Sendo que o mesmo procedimento pode ser feito para a entropia, energias interna ou qualquer outra propriedade termodinâmica:

$$
\begin{aligned}
H\left(T_{1}, P_{1}\right)-H\left(T_{2}, P_{2}\right)= & {\left[H\left(T_{2}, P_{2}\right)-H^{i g}\left(T_{2}, P_{2}\right)\right]+\left[H^{i g}\left(T_{2}, P_{2}\right)-H^{i g}\left(T_{1}, P_{1}\right)\right]-} \\
& {\left[H\left(T_{1}, P_{1}\right)-H^{i g}\left(T_{1}, P_{1}\right)\right] }
\end{aligned}
$$

\subsection{Equação Cúbica de Estado de Peng-Robinson}

Qualquer equação que relacione pressão, temperatura e volume específico de uma substância é chamada de equação de estado. A equação de estado mais simples e mais conhecida é a Equação do Gás Ideal, onde o gás se encontra nas condições de baixas pressões e altas temperaturas. Porém, essa equação é bastante simples e limitada, sendo necessária uma busca por outras equações que representem o comportamento PVT das substâncias com precisão em uma região maior e sem limitações (Çengel e Boles, 2006). Essas equações são chamadas de Equações Cúbicas de Estado.

A equação cúbica de estado de Peng-Robson (Peng e Robinson, 1976) é aplicada principalmente para equilíbrio líquido-vapor. A representação de equilíbrio líquido-vapor é fortemente influenciada pela representação mais precisa da pressão do vapor, que esta implícita na inclusão do fator acêntrico. Uma vez que o ponto crítico e o fator acêntrico caracterizam a pressão de vapor com bastante precisão, a Equação de Peng-Robson também representará com precisão a pressão de vapor (Elliott e Lira, 2012). A EdE de Peng-Robson é dada pela seguinte representação $\mathrm{P} \rho \mathrm{T}$ : 


$$
P=\frac{1}{(1-\rho)}-\frac{a \rho}{R T\left(1+2 b \rho-b^{2} \rho^{2}\right)}
$$

onde a e b são as chamadas constantes da equação de Peng-Robinson e dependem das propriedades críticas como mostrado na Equação 3:

$$
a=\frac{0.45724 R^{2} T_{c}^{2} \alpha(T)}{P_{c}} \quad, \quad b=\frac{0.07780 R T_{c}}{P_{c}}
$$

$\mathrm{Na}$ Equação 3 temos que R é a constante universal dos gases, $T_{c}$ é a temperatura crítica, $P_{c}$ é a pressão crítica e $\alpha(T)$ é uma constante dependente do fator acêntrico $\left(\mathrm{w}_{\mathrm{i}}\right)$ e da temperatura reduzida $\left(T_{r, i}\right)$, podendo ser calculada da seguinte forma:

$$
\alpha(T)=\left[1+m_{i}\left(1-\sqrt{T_{r, i}}\right)\right]^{2} \quad, \quad m_{i}=0.37464+1.54226 w_{i}-0.26992 w_{i}^{2}
$$

A Equação 2 também pode ser representada em termos do fator de compressibilidade, Z, como mostra a Equação 5.

$$
Z=\left[1-\frac{B}{Z}\right]^{-1}-\frac{A}{Z}\left[1+2 \frac{B}{Z}-\left(\frac{B}{Z}\right)^{2}\right]^{-1}
$$

Reorganizando a EdE de Peng-Robinson, é obtida uma função cúbica em termos de Z:

$$
Z^{3}-(1-B) Z^{2}+\left(A-3 B^{2}-2 B\right)-\left(A B-B^{2}-B^{3}\right)=0
$$

As constantes A e B (adimensionais), variam em função das constantes a e b, e podem ser calculadas da seguinte maneira:

$$
A=\frac{a P}{R^{2} T^{2}} \quad, \quad B=\frac{b P}{R T}
$$

Quando da função cúbica da Equação 6 é obtida uma raiz real e duas raízes complexas, a raiz real representa que a substância está no estado de líquido comprimido ou vapor superaquecido, onde para ser vapor superaquecido o valor de $Z$ tem que ser próximo de 1 . Quando são obtidas três raízes reis, a maior raiz representa o vapor saturado e a menor representa o líquido saturado, descartando-se a raiz do meio, pois não possui nenhum significado físico.

\subsection{Ciclo termodinâmico de Potência a vapor}

O ciclo termodinâmico de potência a vapor escolhido neste trabalho é o ciclo Rankine. Este ciclo opera de tal maneira que o fluido de trabalho (água) apresenta uma mudança de fases: líquido comprimido - vapor superaquecido - liquido+vapor saturado - líquido saturado. Uma das características do ciclo Rankine é que a bomba que possui o ciclo rankine 
necessita de pouco trabalho para fornecer água à alta pressão para a caldeira. (Potter e Scott, 2006). A eficiência térmica do ciclo Rankine é calculada pela expressão:

$$
\eta=\frac{W_{t}-W_{b}}{Q_{h}}
$$

Na Equação 8, Wt é o trabalho real fornecido pela turbina, $W b$ é o trabalho que precisa a bomba e $Q h$ é calor fornecido à caldeira.

\section{METODOLOGIA DE CÁLCULO}

Primeiramente foram calculados as Funções de Partida para a entalpia e entropia. Para isso foram feitos os equacionamentos das funções de partida $\mathrm{H}-\mathrm{H}^{\mathrm{ig}}$ e $\mathrm{S}-\mathrm{S}{ }^{\mathrm{ig}}$, até chegar às equações:

$$
\begin{aligned}
& \frac{H-H^{i g}}{R T}=\int_{0}^{\rho}-T\left[\frac{\partial Z}{\partial T}\right] \frac{d \rho}{\rho}+(Z-1) \\
& \frac{S-S^{i g}}{R}=\int_{0}^{\rho}\left[-T\left[\frac{\partial Z}{\partial T}\right]-(Z-1)\right] \frac{d \rho}{\rho}+\operatorname{Ln} Z
\end{aligned}
$$

Usando a EdE de Peng-Robinson descrita na Equação 2, pode-se transformar as Equações 9 e 10 em funções de partida da seguinte maneira:

$$
\begin{gathered}
\frac{H-H^{i g}}{R T}=Z-1-\operatorname{Ln}\left[\frac{Z+(1+\sqrt{2}) B}{Z+(1-\sqrt{2}) B}\right] \frac{A}{B \sqrt{8}}\left[1+\frac{K \sqrt{T_{r}}}{\sqrt{\alpha}}\right] \\
\frac{S-S^{i g}}{R}=\operatorname{Ln}(Z-B)-\operatorname{Ln}\left[\frac{Z+(1+\sqrt{2}) B}{Z+(1-\sqrt{2}) B}\right] \frac{A}{B \sqrt{8}}\left[\frac{K \sqrt{T_{r}}}{\sqrt{\alpha}}\right]
\end{gathered}
$$

Após isso foi escolhido um ciclo Rankine simples mostrado na Figura 1 para fazer o cálculo da eficiência térmica a partir das funções de partida obtidas anteriormente. Para obter a eficiência foi calculado separadamente cada constituinte do ciclo começando pela turbina, onde se obteve o trabalho produzido, Wt. Em seguida se obteve o trabalho que precisa a bomba, $W b$, finalizando com o calor fornecido à caldeira, $Q h$. Com esses valores foi possível calcular a eficiência da Equação 8.

\section{RESULTADOS E DISCUSSÕES}

\subsection{Obtenção dos valores tabelados}

Para o cálculo das funções de partida foram necessários conhecer as propriedades da água: pressão e temperatura crítica, fator acêntrico e massa molar, além das constantes de calor específico para gás ideal. Esses dados são mostrados na Tabela1. 
Figura 1 - Ciclo Rankine.

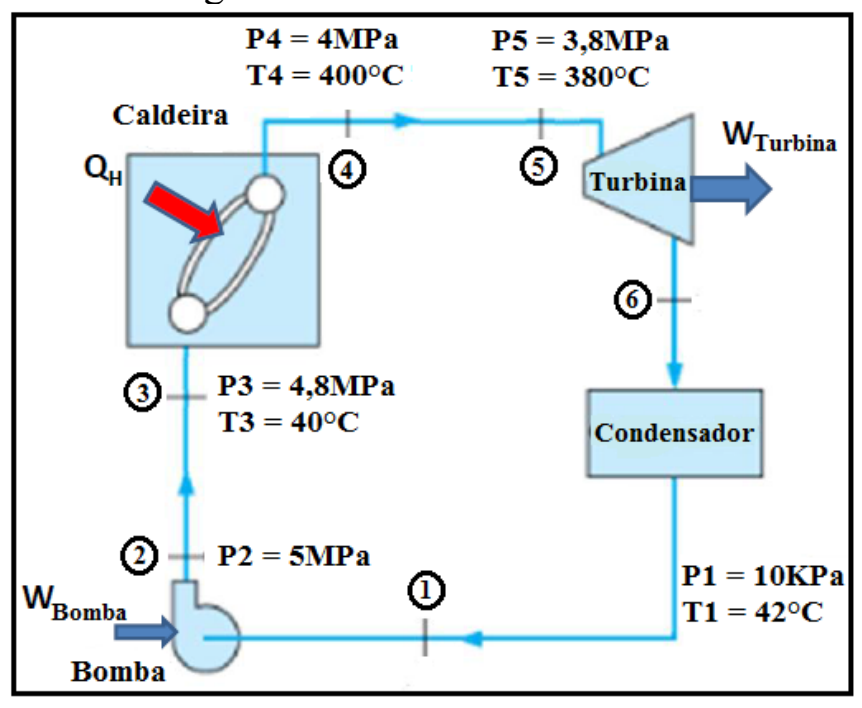

Tabela 1 - Propriedades críticas, fator acêntrico e constantes do calor específico a pressão constante da água.

\begin{tabular}{cc}
\hline Tc $(\mathrm{K})$ & 647,3 \\
$\mathrm{Pc}(\mathrm{MPa})$ & 22,09 \\
$\omega$ & 0,345 \\
Massa molar $(\mathrm{g} / \mathrm{mol})$ & 18,0 \\
\hline Constantes: $\mathrm{Cp}=\mathrm{A}+\mathrm{BT}+\mathrm{CT}^{2}+\mathrm{DT}^{3}$ \\
\hline A & 32,24 \\
$\mathrm{~B}$ & 0,001924 \\
$\mathrm{C}$ & 0,00001055 \\
$\mathrm{D}$ & $3,215 \mathrm{E}-08$ \\
\hline
\end{tabular}

$\mathrm{Cp}(\mathrm{J} / \mathrm{mol} . \mathrm{K}) ; \mathrm{T}(\mathrm{K})$.

\subsection{Cálculo da eficiência do Ciclo de Rankine}

Primeiramente calculou-se o trabalho realizado pela turbina. Para isto, sabendo que a turbina realiza uma expansão isentrópica, e fazendo os balanços de massa, energia e entropia pode-se chegar a dois equacionamentos utilizando a Equação 1:

$$
\begin{aligned}
& \left\lfloor S\left(T_{2}, P_{2}\right)-S^{i g}\left(T_{2}, P_{2}\right)\right\rfloor+\left\lfloor S^{i g}\left(T_{2}, P_{2}\right)-S^{i g}\left(T_{1}, P_{1}\right)\right\rfloor-\left\lfloor S\left(T_{1}, P_{1}\right)-S^{i g}\left(T_{1}, P_{1}\right)\right\rfloor=0 \\
& {\left[H\left(T_{2}, P_{2}\right)-H^{i g}\left(T_{2}, P_{2}\right)\right]+\left[H^{i g}\left(T_{2}, P_{2}\right)-H^{i g}\left(T_{1}, P_{1}\right)\right]-\left[H\left(T_{1}, P_{1}\right)-H^{i g}\left(T_{1}, P_{1}\right)\right]=W t(14)}
\end{aligned}
$$

Nas Equações 13 e 14, o estado termodinâmico $1\left(\mathrm{~T}_{1}, \mathrm{P}_{1}\right)$ representa as propriedades de entrada da turbina e o estado $2\left(\mathrm{~T}_{2}, \mathrm{P}_{2}\right)$ as propriedades de saída. Como o valor de $\mathrm{T}_{2}$ é desconhecido, pela Equação 13 se pode calcular $\mathrm{T}_{2}$ às condições isentrópicas. Utilizando esse valor na Equação 14 se pode obter o trabalho gerado pela turbina. Este método também foi utilizado para calcular o trabalho fornecido pela bomba. Já o calor fornecido pela caldeira utilizou-se apenar a Equação 14, que no lugar do Wt foi calculado o Qh, como demostrado 
pela primeira lei da termodinâmica. A Tabela 2 apresenta os resultados obtidos das funções de estado de entalpia $(\mathrm{J} / \mathrm{mol})$ e entropia $(\mathrm{J} / \mathrm{mol} . \mathrm{K})$ e também os resultados gerados pelo software ChemSOF.

Tabela 2 - Valores obtidos e valores gerados pelo ChemSOF das funções de estado.

\begin{tabular}{c|ccc|ccc}
\hline \multirow{2}{*}{$\begin{array}{c}\text { Funções de } \\
\text { Estado }\end{array}$} & \multicolumn{2}{|c|}{ Resultados obtido neste trabalho } & \multicolumn{3}{|c}{ Resultado fornecido pelo ChemSOF } \\
\cline { 2 - 7 } & Turbina & Bomba & Caldeira & Turbina & Bomba & Caldeira \\
\hline$\left(\mathrm{S}_{1}-\mathrm{S}_{1}{ }^{\mathrm{ig}}\right)$ & $-1,28$ & $-139,88$ & - & $-1,28$ & $-139,77$ & - \\
\hline$\left(\mathrm{H}_{1}-\mathrm{H}_{1}{ }^{\mathrm{ig}}\right)$ & $-1181,97$ & $-44973,67$ & $-45030,88$ & $-1183,75$ & $-44924,14$ & $-44938,66$ \\
\hline$\left(\mathrm{S}_{2}-\mathrm{S}_{2}{ }^{\mathrm{ig}}\right)$ & $-0,06$ & $-88,29$ & - & $-0,06$ & $-88,05$ & - \\
\hline$\left(\mathrm{H}_{2}-\mathrm{H}_{2}{ }^{\mathrm{ig}}\right)$ & $-18,94$ & $-44893,19$ & $-1176,95$ & $-18,96$ & $-44802,67$ & $-1178,75$ \\
\hline
\end{tabular}

Nota-se que os valores para as funções de estado da entalpia como da entropia ficaram próximos aos valores fornecidos pelo software comercial. A eficiência do ciclo foi de $24,89 \%$ e a eficiência calculada através das tabelas termodinâmicas foi de 29,40\%. Comparando os resultados observa-se que o valor obtido através das funções de partida usando a EdE de Peng-Robinson foi próximo ao valor obtido com as tabelas termodinâmicas.

\section{CONCLUSÃO}

Verificou-se neste trabalho que as funções de partida usadas junto com as EdEs são recursos eficazes para o cálculo de propriedades termodinâmicas como entalpia e entropia, que não possuem valores absolutos e mensuráveis, além de ser usadas para fluídos complexos que não possuem valores tabelados. Os valores das funções de partida da entropia e entalpia e a eficiência do ciclo de potência a vapor apresentaram resultados razoáveis e próximos aos apresentados pelo ChemSOF e tabelas termodinâmicas, comprovando-se essa eficiência.

\section{REFERÊNCIAS BIBLIOGRÁFICAS}

ChemSOF, Tips \& Tricks for Chemical Engineers. www.chemsof.com.

ÇENGEL, Y.A.; BOLES, M. A. Termodinâmica. Quinta edição. Editora McGraw-Hill., 2006.

ELLIOTT, J.R.; LIRA, C.T. Introductory Chemical Engineering Thermodynamics. Segunda edição. Editora Prentice Hall, 2012.

PENG, D.Y.; ROBINSON, D.B. A new two constant equation of state. Ind. Eng. chem. Fundam., v. 15, p. 59-64, 1976.

PRAUSNITZ, J. M.; POLING, B. C.;O’CONNELL, J. P. The Properties of Gases \& Liquids. Quarta edição. Editora McGraw-Hill., 2001.

POTTER, M.C.; SCOTT, E.P. Termodinâmica. Editora Thomson, 2006. 